\title{
Modeling Three-Dimensional Geometric Shapes from Nucleic Acid Sequences Using a Computerized Numerical Control
}

\author{
Bandar Ali Suliman \\ Department of Medical Laboratory Technology, College of Applied Medical Sciences, Taibah University, Madinah, Saudi Arabia \\ Email address: \\ bsuliman@taibahu.edu.sa
}

\section{To cite this article:}

Bandar Ali Suliman. Modeling Three-Dimensional Geometric Shapes from Nucleic Acid Sequences Using a Computerized Numerical Control. International Journal of Clinical and Experimental Medical Sciences. Vol. 5, No. 3, 2019, pp. 49-52. doi: 10.11648/j.ijcems.20190503.12

Received: July 14, 2019; Accepted: August 5, 2019; Published: August 16, 2019

\begin{abstract}
The nucleic acid sequence is an astonishing and a complicated coding system that is capable of producing a complete human body with of its molecules, cells, tissue and organs. Nucleic acid has been used in many fields of sciences for the preservation and encoding of different types of information. The current project describes the use of a computerized numerical control device to form 3D geometric shapes from nucleic acid sequences. The device employs dynamic algorithms to store and then identify the strings of letters of the nucleic acid sequence, transforming them into trigonometric matrices of continues triangular codes and ultimately translating each one of matrix codes into points in 3D space to construct three-dimensional geometric shapes. This method is useful for storing architectural design and blueprints, as well as, helping to establish a standardized coding technology for 3D printing devices.
\end{abstract}

Keywords: DNA Sequence, 3D Structure, Medical Coding

\section{Introduction}

The nucleic acid sequence is an astonishing and a complicated coding system that is capable of producing a complete human body with of its molecules, cells, tissue and organs. Since the discovery and subsequent description of the nature of the DNA sequence [1, 2], many molecular biologists were intrigued to understand the ability of a particular DNA sequence to produce a functional protein with a unique $3 \mathrm{D}$ structure $[3,4]$.

Many scientists tried to use the nucleic acid sequence to alter the geometric shape or other physical properties of constructed materials [5-7]. Through scientific developments and the introduction of 3D printing technology, other scientists were able to demonstrate the successful transformation of nucleic acid sequences into a transferable and reproducible shapes [8, 9]. In 2009, a Chinese patent was registered to demonstrate the ability of transforming the nature and depth of cryptographic images through the two-dimensional chaotic sequence (or the primary coding) into a code of DNA sequence as the final output [10]. Later, another Chinese patent implemented and subsequently developed the theory that was used in the prior description of the nature and depth of cryptographic images to reduce the coefficient of change and standard deviation in the mathematical outputs of two-dimensional outputs [11].

Recently, DNA has been used in the preservation and encoding of multiple types of information, ranging from ordinary personal computer files with any extensions [12], to digital productions files aimed for long-term storage [13]. This recent biotechnological science has been considered recently as a new area and has occupied the scientist concern on how to use DNA for storing and encrypting information and data with a standardized format that can be widely distributed in larger scales across the biotechnology industry [14-16].

In this project, we introduced a novel method to store and transfer engineering drawings composed for 3D structures and constructs through nucleic acid sequences that can be stored in an identical and standard manner which is similar in all countries and in all languages and despite of the difficult environmental and climatic storage conditions.

\section{Methods}

\subsection{The Primary Coding System}

Since the DNA sequence is based on four probabilities for each nucleotide (Table 1), and by assuming fifteen nucleotide positions are treated as a single entity; the number of 
mathematical permutations of DNA sequences (primary coding) can be as follows (1):

$$
4^{\wedge 15}=1,073,741,824
$$

Table 1. The four different types of nucleobases that builds up a DNA sequence.

\begin{tabular}{llll}
\hline Nucleobases & & & \\
\hline $\mathrm{A}$ & $\mathrm{T}$ & $\mathrm{C}$ & $\mathrm{G}$ \\
\hline
\end{tabular}

\subsection{The Trigonometric (Secondary) Coding System}

The trigonometric depends mainly on the coding unit that we have described earlier. Using the previously patented coding unit, it is applicable to obtain five different variables for each coding unit which then can be encrypted for each axis; positive and negative. By assuming thirteen nucleotide positions are treated as a single entity; the number of mathematical permutations of trigonometric coding unit (secondary code) can be as follows (2):

$$
5^{\wedge 13}=1,220,703,125
$$

\subsection{Final Output Dimensioning System}

Three dimensional geometric shapes are defined by three different axes including a positive and negative direction for each one of the three dimensions. Therefore, the number of variables which is required to determine any point in $3 \mathrm{D}$ space can be calculated, when considering the scale is $1 \mathrm{~mm}$ and the total geometric dimensions are $20 \mathrm{~cm} \times 20 \mathrm{~cm} \times 20 \mathrm{~cm}$, as follows (3):

$$
\text { Location }=(000.000 .000)
$$

Adding the basic concept of Boolean operations [17] aids the conversion of permutations from cubic diagrams to chaotic points that generate non-standard geometric shapes such as cones and domes. Therefore, Lorenz equations [18] can be used to calculate the mathematical integrals of dimensions for width and length, where the equation can be as follow (4):

$$
\Delta \mathrm{y} / \Delta \mathrm{x}=\int(\mathrm{x}+\Delta \mathrm{x})-\int(\mathrm{x}) / \Delta \mathrm{x}
$$

When the third dimension (height) of mathematical integrations is introduced, the equation will become as follows (5):

$$
\iiint_{\mathrm{R}}=(\mathrm{x} \cdot \mathrm{y} \cdot \mathrm{z}) \Delta \mathrm{V}
$$

\subsection{The Formation of Triangular Matrix Arrays}

The next step is forming longitudinal and transverse matrices that are constructed based on triangular coding (Equation 2) which depends on the size of the geometric shape to be formed. Then, a complete set of triangular matrix arrays containing thirteen triangular encoders that can be added for each additional $8 \mathrm{~cm}$ of the geometry size to be configured with a drawing factor equal to $1 \mathrm{~mm}$.

\subsection{Coding Information}

Triangular matrix arrays code information derived from
DNA sequences that is associated with mathematical equations that can be measured using Equation (1). DNA sequences can be encoded into trigonometric (secondary coding) and then to the points which is forming the geometric shape in the three basic dimensions according to the example shown in the following table (Table 2).

Table 2. The conversion of the DNA sequence (the primary code) to the trigonometric variables (the secondary code) and that can be translated using the algorithms into points in $3 D$ space (the final output) by adding different mathematical levels in a form of an array.

\begin{tabular}{ll}
\hline Encoding & The Real Variables \\
\hline Primary & ATG CCG TAT CGT ATA GTC \\
Secondary & $(018 .-103.877)$ \\
Final output & \\
\hline
\end{tabular}

\subsection{The Computerized Numerical Control}

The computerized numerical control (CNC) contains a processing unit that is capable of identifying DNA sequences according to internationally recognized symbols and then encode them as dynamic variables into a triangular encoding. The final output of the coding information will then be converted to model the spatial points in 3D space, according to the three dimensions, and then form the final geometric shape to be manufactured or synthesized using longitudinal, temporal and vertical movement.

\section{Results}

We used a computerized numerical control device to construct 3D geometric shapes from nucleic acid sequences. First, the computerized numerical computer control was designed to contain a computer processing unit that employs a non-removable medium in which the coding system, containing the schematic diagram of primary and secondary coding, is stored (Figure 1). The processing unit is configured to identify the DNA sequences, entered by the user, and then is transformed into a 3D structure through a series of geometric algorithms.

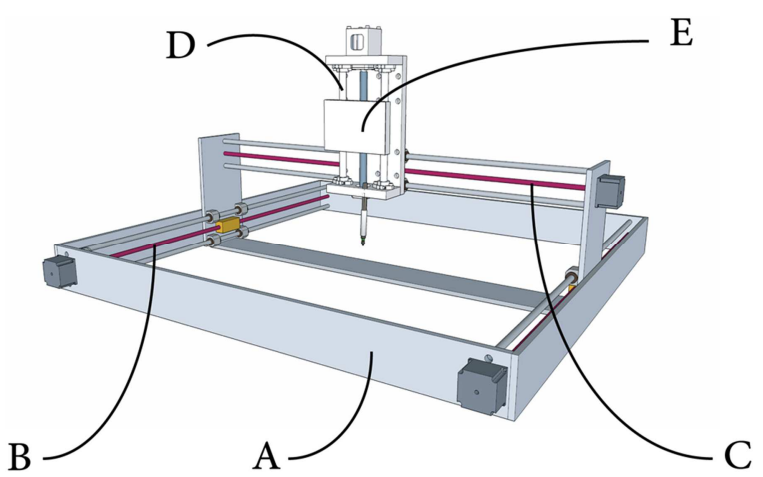

Figure 1. The computerized numerical computer device. A) The external structural framework of the numerical control device; $B$ ) The axis of the longitudinal movement [X axis] of the device; $C$ ) The axis of the lateral movement [Y axis] of the device; D) The axis of the vertical movement [ $Z$ axis] of the device; E) The computer processing unit containing a non-removable media storage medium. 
The DNA sequence is composed of four different bases as shown in (Table 1) composing the primary code in which the shape is stored. In this project, we employed a new methodology to transform the trigonometric secondary coding system into points forming a geometric shape by using a series of formulas (Formula 1-5). Next, the method of forming the geometric dimensions of the three-dimensional shapes using multiple levels of axes is presented. Using this method, the length, width, and height of the desired shape can be calculated into the 3D space of a hypothetical cube. The configuration of the cubic scheme relies on the three-dimensional geometry that needs to be modeled. Figure 2 demonstrates the method of calculating positive and negative dimensions from the intersection of the three axes with each other, thus, each of the three dimensions has two hypotheses: positive and negative. The cubic scheme consists of the three main axes. The positive longitude (or the $\mathrm{X}+$ axis) is the direction to the right of the intersection of the three main axes, and the negative longitude (or the $\mathrm{X}$ - axis) is the direction to the left of the junction of the three main axes. The positive vertical direction (or the $\mathrm{Y}+$ axis) is the direction from the highest point of the three main axes, as well as the negative vertical direction (or the $\mathrm{Y}$ - axis). The positive cross-direction (or the $\mathrm{Z}+$ axis) is the direction above of the intersection of the three main axes, and the negative cross-direction (or the Z-axis) is under the intersection of the three main axes.

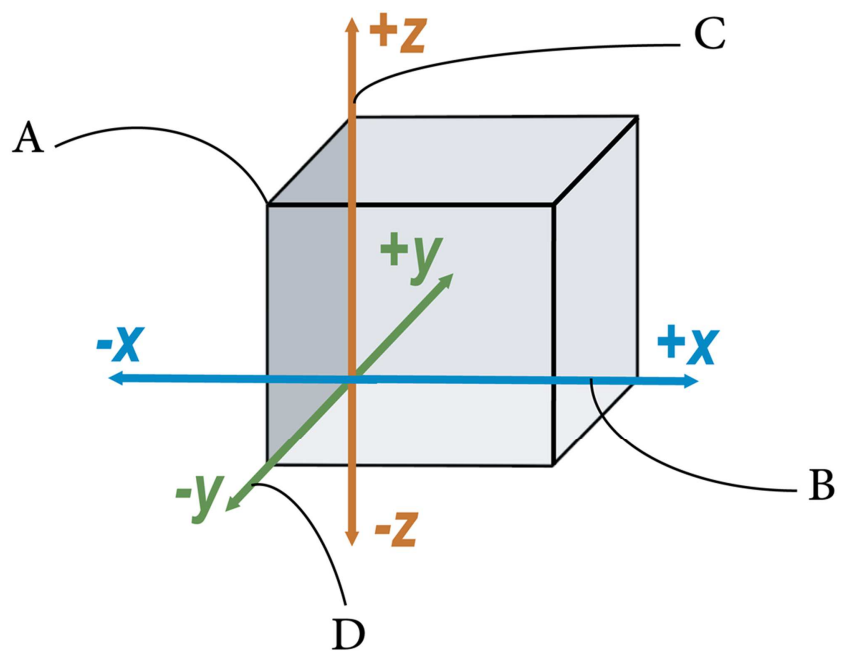

Figure 2. Building the basic geometric shape in $3 D$ space. A) the basic structure encompassing the $3 D$ space for all three axes; B) longitudinal axis; C) vertical axis; D) cross-direction axis.

The idea of transforming DNA sequence into an intermediary coding system based on triangles was described by us before [19]. In this project the DNA sequence variables was converted into five different variables that are then used by the mathematical levels (Figure 3). After the formation of mathematical levels, which are based on the three-dimensional plan of the diagrams, the coefficient of rotation and deviation of each of the three main dimensions is added to the final geometric shape.
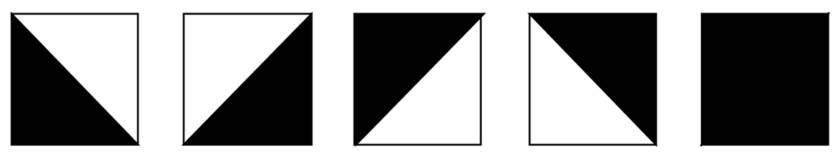

Figure 3. The five different variables for DNA sequence used in this project.

Equation (5) is then used to calculate any point in the space according to the theory of mathematical chaos. This step marks the transformation from actual DNA sequence to a coding system through the final output dimensioning system. The triangular matrix arrays are then formed storing the actual points of the designated 3D shape into $\mathrm{X}, \mathrm{Y}$ and $\mathrm{Z}$ coordinates (Figure 4).

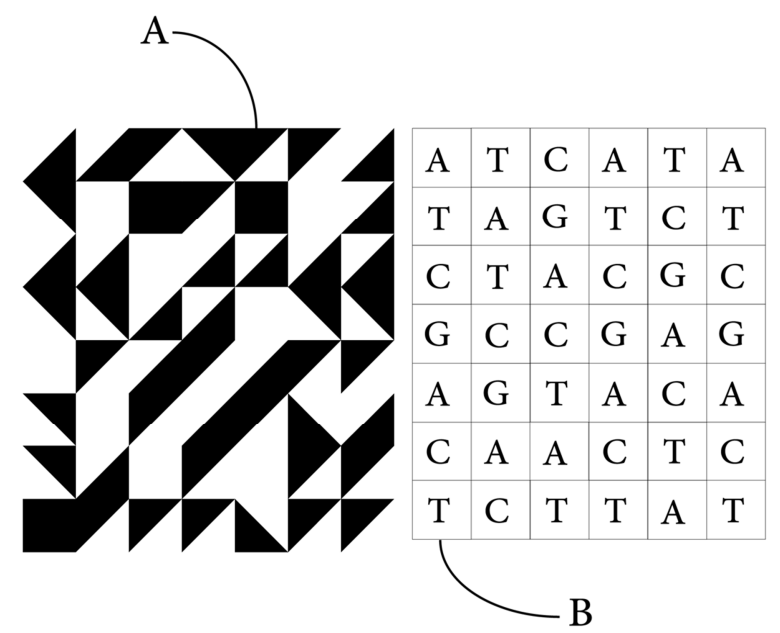

Figure 4. A triangular matrix of arrays containing the DNA variables that are transformed by the secondary coding system. A) the triangular matrix; B) the $D N A$ sequence before the conversion process.

The computerized numerical control is then able to target each point in space since the required dimensions are identified from the triangular matrix. These points are scattered into a cubic shape (Figure 5) to facilitate the formation of each designated shape or output by the CNC.

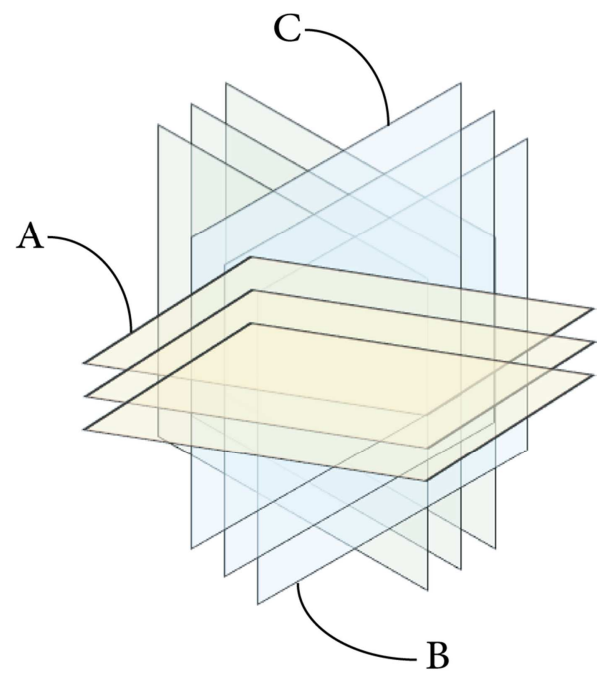

Figure 5. The cubic structure containing all the necessary points which are required by the CNC to formulate the final geometric shape from the triangular matrix using arrays of mathematical levels. A) X plains; B) $Y$ plains; C) Z plains. 


\section{Discussion}

In the current innovation, the numerical control system can identify the strings of nucleic acids to convert the extracted code into three-dimensional geometric shapes. At the numerical control device, the numerical processing unit is able to identify deoxyribonucleic acid (primary coding) and transform each single nucleic acid from the DNA sequence into trigonometric coding variables (secondary coding). This process is performed by employing variable dynamic algorithms which assist the numerical control device in generating the three basics dimensions (length, width, height). Then, the trigonometric coding is converted from graphical map into visual graphics, representing the geometry (final output). This novel method will provide a new mechanism by which DNA sequences is converted into secondary coding, that is not based on binary dimension as mentioned in previous patents. This method will also be useful for storing architectural design and blueprints, as well as, helping to establish a standardized coding technology for 3D printing devices. However, the secondary coding of DNA sequences using the current novel method is converted into three-dimensional geometric shapes using Triangle coding system which depends on mathematical chaos theory-based dynamic algorithm.

\section{Conclusion}

DNA is in the center of importance because it holds the genetic code to life. Cells use the DNA sequence as a genetic code to store biological information leading to the production of proteins with specific and unique 3D shapes. Here we present a novel method to store and transfer blueprints for 3D structures and shapes through nucleic acids which can be stored in an identical and standard manner that is similar in all countries and in all languages and despite of the difficult environmental and climatic conditions. This method will pave the way to more advanced and detailed technologies in the field of molecular fabrication and assembly.

\section{Acknowledgements}

The authors would like to thank Ms. Nabila Qadri (Director, Masheej Medical LLC) for her support and encouragement throughout the preparation of this manuscript.

\section{Conflict of Interest}

This work has been patented in Saudi Arabia through King Abdul-Aziz City for Science and Technology (IPC/SPO 6100).

\section{References}

[1] Wang, A. H.-J., et al., Molecular structure of a left-handed double helical DNA fragment at atomic resolution. Nature, 1979. 282 (5740): p. 680.
[2] Soutourina, J., Transcription regulation by the Mediator complex. Nature reviews Molecular cell biology, 2018. 19 (4): p. 262.

[3] Drew, H. R., et al., Structure of a B-DNA dodecamer: conformation and dynamics. Proceedings of the National Academy of Sciences, 1981.78 (4): p. 2179-2183.

[4] Tokura, Y., et al., Fabrication of Defined Polydopamine Nanostructures by DNA Origami - Templated Polymerization. Angewandte Chemie International Edition, 2018. 57 (6): p. 1587-1591.

[5] Wang, Z., et al., Discovery of the DNA "genetic code" for abiological gold nanoparticle morphologies. Angewandte Chemie International Edition, 2012. 51 (36): p. 9078-9082.

[6] Zhou, W., et al., Design Rules for Template - Confined DNA Mediated Nanoparticle Assembly. Small, 2018. 14 (44): p. 1802742 .

[7] Shahbazi, M. A., T. Bauleth - Ramos, and H. A. Santos, DNA hydrogel assemblies: Bridging synthesis principles to biomedical applications. Advanced Therapeutics, 2018. 1 (4): p. 1800042.

[8] Loo, A. H., C. K. Chua, and M. Pumera, DNA biosensing with 3D printing technology. Analyst, 2017. 142 (2): p. 279-283.

[9] Wang, L., et al., Synthetic genomics: from DNA synthesis to genome design. Angewandte Chemie International Edition, 2018. 57 (7): p. 1748-1756.

[10] Qiang, Z., X. Xianglian, and W. Xiaopeng, Digital image encryption method based on DNA sequence and multi-chaotic mapping, in Baidu Zhuanli, National Intellectual Property Administration, Editor. 2009: China.

[11] Xin, J., et al., Image encryption method based on information entropy and a spatiotemporal chaotic system, in Baidu Zhuanli, National Intellectual Property Administration, Editor. 2015: China.

[12] Goldman, N., et al., Towards practical, high-capacity, low-maintenance information storage in synthesized DNA. Nature, 2013. 494 (7435): p. 77.

[13] Cox, J. P., Long-term data storage in DNA. TRENDS in Biotechnology, 2001. 19 (7): p. 247-250.

[14] Liu, C., et al., DNA barcode goes two-dimensions: DNA QR code web server. PloS one, 2012.7 (5): p. e35146.

[15] Donohoue, P. D., R. Barrangou, and A. P. May, Advances in industrial biotechnology using CRISPR-Cas systems. Trends in Biotechnology, 2018. 36 (2): p. 134-146.

[16] Katz, L., et al., Synthetic biology advances and applications in the biotechnology industry: a perspective. Journal of industrial microbiology \& biotechnology, 2018. 45 (7): p. 449-461.

[17] Rappoport, A. and S. Spitz. Interactive boolean operations for conceptual design of 3-d solids. in Siggraph. 1997.

[18] Sparrow, C., The Lorenz equations: bifurcations, chaos, and strange attractors. Vol. 41. 2012: Springer Science \& Business Media.

[19] Suliman, B. A., A two-dimensional triangular medical coding system for storing and reporting medical information. Biomedical Research, 2019. 30 (4): p. 521-523. 